\title{
Core and rod structures of a thermophilic cyanobacterial light-harvesting phycobilisome
}

2 Keisuke Kawakami ${ }^{1} \dagger^{*}$, Tasuku Hamaguchi ${ }^{1} \dagger$, Yuu Hirose $^{2}$, Daisuke Kosumi ${ }^{3}$, Makoto Miyata ${ }^{4}$,

3 Nobuo Kamiya ${ }^{5}$, Koji Yonekura ${ }^{1,6,7 *}$

$4 \quad{ }^{1}$ Biostructural Mechanism Laboratory, RIKEN SPring-8 Center, 1-1-1 Kouto, Sayo; Hyogo 679-

5 5148, Japan.

$6 \quad$ 2Electronics-Inspired Interdisciplinary Research Institute, Toyohashi University of Technology;

7 Aichi 441-8580, Japan.

$8 \quad{ }^{3}$ Institute of Industrial Nanomaterials, Kumamoto University; Kumamoto 860-8555, Japan.

$9 \quad{ }^{4}$ Graduate School of Science, Osaka City University; Osaka 558-8585, Japan.

$10 \quad{ }^{5}$ The OCU Advanced Research Institute for Natural Science \& Technology (OCARINA), Osaka

11 City University; Osaka 558-8585, Japan.

$12{ }^{6}$ Advanced Electron Microscope Development Unit, RIKEN-JEOL Collaboration Center,

13 RIKEN Baton Zone Program; Hyogo 679-5148, Japan.

$14 \quad{ }^{7}$ Institute of Multidisciplinary Research for Advanced Materials, Tohoku University; Miyagi

15 980-8577, Japan.

16 *Corresponding authors:

17

18 Keisuke Kawakami

19 Biostructural Mechanism Laboratory

20 RIKEN SPring-8 Center, 1-1-1 Kouto

21 Sayo, Hyogo 679-5148, Japan 
Tel: $+81-0791-58-0802$

Koji Yonekura

Biostructural Mechanism Laboratory

RIKEN SPring-8 Center, 1-1-1 Kouto

Sayo, Hyogo 679-5148, Japan

E-mail: yone@spring8.or.jp

Tel: +81-0791-58-0802

Summary: Cyanobacteria, glaucophytes, and rhodophytes utilize giant, light-harvesting phycobilisomes (PBSs) for capturing solar energy and conveying it to photosynthetic reaction centers. PBSs are compositionally and structurally diverse, and exceedingly complex, all of which pose a challenge for a comprehensive understanding of their function. To date, three detailed architectures of PBSs by cryo-electron microscopy (cryo-EM) have been described: a hemiellipsoidal type, a block-type from rhodophytes, and a cyanobacterial hemidiscoidal-type. Here, we report cryo-EM structures of a pentacylindrical allophycocyanin core and phycocyanin-containing rod of a thermophilic cyanobacterial hemidiscoidal PBS. The structures define the spatial arrangement of protein subunits and chromophores, crucial for deciphering the energy transfer mechanism. They reveal how the pentacylindrical core is formed, identify key interactions between linker proteins and the bilin chromophores, and indicate pathways for unidirectional energy transfer. 


\section{Introduction}

Cyanobacteria, glaucophytes, and rhodophytes utilize a large water-soluble, light-harvesting complex called phycobilisome (PBS) for solar energy absorption and energy transfer to photosynthetic membrane proteins (photosystem I and photosystem II; PSI and PSII) ${ }^{1}$. PBSs absorb light in the wavelength range 490-650 nm (as an exception, cyanobacteria containing chlorophyll f have PBSs that absorb near-infrared light ${ }^{2}$, which is otherwise difficult for PSI and PSII to harness (Extended Data Fig. 1). PBS is composed of phycobiliproteins (PBPs) such as phycoerythrin, phycoerythrocyanin, phycocyanin (PC), and allophycocyanin (APC). Assembly units of the PBPs are $\alpha$ - and $\beta$-subunits that have globin folds and harbor several linear tetrapyrrole chromophores such as phycoerythrobilin, phycourobilin, phycoviolobilin, and phycocyanobilin $(\mathrm{PCB})^{3}$. Oligomers of these $\alpha$ - and $\beta$-subunits are assembled through nonchromophorylated linker proteins to construct the whole PBS complex. Five types of PBS structural morphology have been reported: hemidiscoidal ${ }^{4,5}$, hemiellipsoidal ${ }^{6}$, block-type $^{7}$ rodtype $^{8,9}$, and bundle-type ${ }^{10}$. Hemidiscoidal PBSs have bicylindrical ${ }^{11}$ tricylindrical $^{4}$, or pentacylindrical cores ${ }^{5}$. Composition of PBPs with respect to number of rods, the overall structure of the PBSs, and their association with PSI and PSII are regulated by availability of light and nutrients ${ }^{9,12}$. Recently, the three-dimensional (3D) structures of three types of red algal PBSs were analyzed by cryo-electron microscopy (cryo-EM) $)^{13-16}$, and details of their tricylindrical cores and energy transfer pathways were revealed. Since PBSs are compositionally and structurally diverse and exceedingly complex, structural and functional analyses of PBSs in different species are essential for comprehensive understanding of their working mechanisms.

The PBS of Thermosynechococcus vulcanus NIES-2134 (hereafter referred to as T. vulcanus) has a hemidiscoidal structure with a pentacylindrical APC core and six PC rods, the total 
molecular weight of which reaches a few MDa or more ${ }^{16,17}$ (Extended Data Fig. 2). These are composed of $\alpha-(\mathrm{CpcA}, \mathrm{ApcA}, \mathrm{ApcD}$, and $\mathrm{ApcE})$ and $\beta-(\mathrm{CpcB}, \mathrm{ApcB}$, and $\mathrm{ApcF})$ subunits, and their basic constituent unit is an $\alpha \beta$ monomer ${ }^{18}$. PCBs are covalently bound via a thio-ether linkage to a conserved cysteine residue in the $\alpha$ and $\beta$ subunits ${ }^{19}$. Three PC monomers (trimer, $\left.(\alpha \beta)_{3}\right)$ paired with two trimers form a disk (hexamer, $(\alpha \beta)_{6}$ ), and two disks interact with each other to form a PC rod; the arrangement and number of PC rods vary among species ${ }^{4,5}{ }^{9}$. Linker proteins in the PC rods are believed to contribute not only to structural stability between the disks but also to adjustment of the energy level of the chromophores. Linker proteins of $T$. vulcanus are classified as rod linker $\left(\mathrm{L}_{\mathrm{R}} ; \mathrm{CpcC}\right)$; rod-terminal linker $\left(\mathrm{L}_{\mathrm{RT}} ; \mathrm{CpcD}\right)$; rod-core linkers ( $\mathrm{L}_{\mathrm{RC}}$; $\mathrm{CpcG1}, \mathrm{CpcG} 2$, and $\left.\mathrm{CpcG} 4\right)$; core-membrane linkers ( $\mathrm{L}_{\mathrm{CM}}$; $\mathrm{ApcE}$ ), which bind the core to thylakoid membranes; or core linkers $\left(\mathrm{L}_{\mathrm{C}} ; \mathrm{ApcC}\right)$, which bind to the PBS core. When the PBS absorbs light, the excitation energy is transferred at a very fast rate (of the order of picoseconds) to chromophores in subunits at the membrane surface called terminal emitters $\left(\mathrm{L}_{\mathrm{CM}}, \mathrm{ApcD}\right.$, and $\left.\mathrm{ApcF}\right)$ and then eventually to PSII and $\mathrm{PSI}^{20}$. Several X-ray crystal structures of PCs and APCs from T. vulcanus have been reported, and these have provided starting points for discussion around possible overall structural configurations and mechanisms of internal energy transfer in PBSs ${ }^{21-27}$.

Here, we report the structures of the pentacylindrical APC core and PC rod from T. vulcanus at resolutions of $3.7 \AA$ and $4.2 \AA$, respectively. The structures reveal the detailed architecture of hemidiscoidal PBSs and indicate a possible mechanism for the unidirectional excited energy transfer. They provide a basis for the understanding of the many cyanobacterial PBSs and could be utilized for applications for the efficient use of solar energy ${ }^{27-29}$. 


\section{Results and Discussion}

\section{Structural refinement and overall structure of PBS core}

The pentacylindrical APC core (PBS core) was purified from preparations of PBSs followed by gradient fixation (GraFix) treatment ${ }^{17,31}$. Most PC rods were absent probably because of dissociation during preparation of the cryo-EM grids (see Materials and Methods). Cryo-EM maps of the PBS core and PC rod were reconstructed to $3.7 \AA$ and $4.2 \AA$ resolutions, respectively, based on the Gold Standard Fourier shell correlation [FSC] criteria of 0.143 between two half maps (Extended Data Table 1). The structural models were refined against the maps, which validated the resolutions; FSC of 0.5 between the model and the map: $3.8 \AA$ (PBS core) and 4.2 $\AA$ (PC rod); Q-score ${ }^{32}$ based estimates: $3.6 \AA(\mathrm{Q}=0.49$; PBS core $)$ and $4.0 \AA(\mathrm{Q}=$ 0.40; PC rod) (Extended Data Figs. 3 and 4, and Extended Data Tables 1-3).

The PBS core of $T$. vulcanus shows a hemidiscoidal structure with $C 2$ symmetry, composed of three cylinders (A, A', and B), two cylinders (C and C'), and PC rods (Fig. 1). The strict twofold symmetry was not held in the outer part of the PC rods (Rb, Rb', Rt, Rt', Rs1, Rs1', Rs2, and Rs $\left.2^{\prime}\right)^{17}$, as many of these parts likely dissociated from the core during sample preparation (see above). The cryo-EM map resolves portions of the rods, but local resolution of the corresponding regions ranges from 7 to $20 \AA$ (Extended Data Fig. 3). Thus, we did not build models of the rods (Fig. 1C). The PBS core is a supercomplex with the dimensions $110 \times 210 \times 300 \AA^{3}$ and composed of 38 ApcAs, 40 ApcBs, 6 ApcCs, 2 ApcDs, $2 \mathrm{~L}_{\mathrm{CMS}}, 2$ ApcFs, and 84 PCBs (Extended Data Fig. 5, Extended Data Table 4). The number of APC trimers in each cylinder of the PBS core differs between species $4,5,13,14,16,17)$, implying that the architecture of a PBS affects the efficiency of energy transfer within the PBS as well as from PBS to PSII/PSI. 

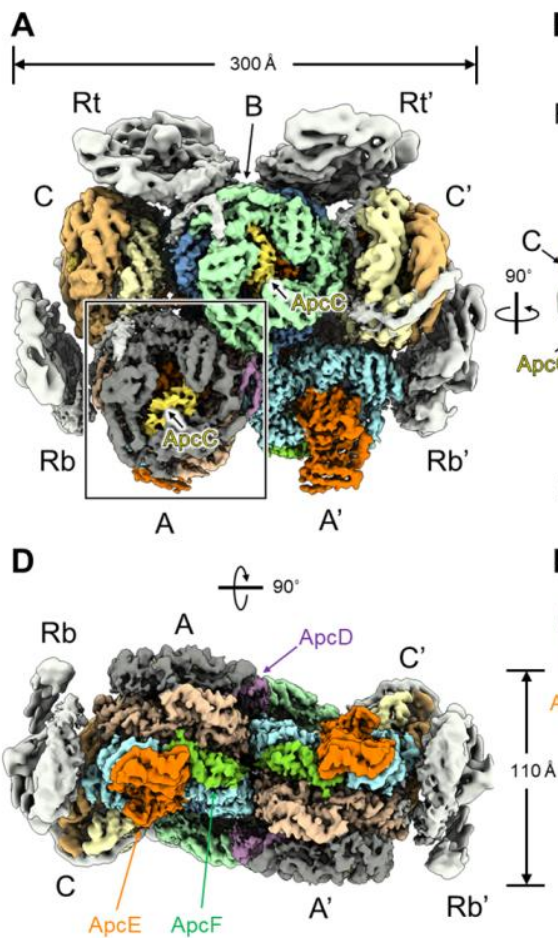

E

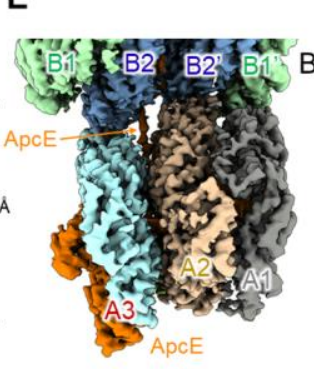

A
C

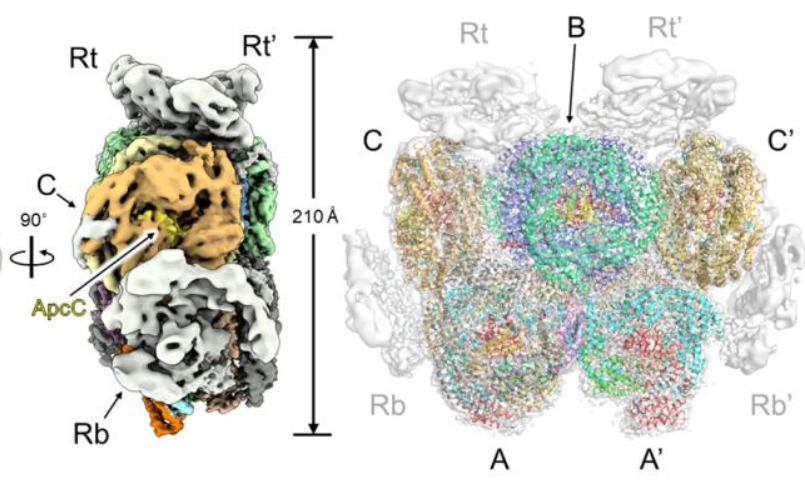

$\mathbf{F}$

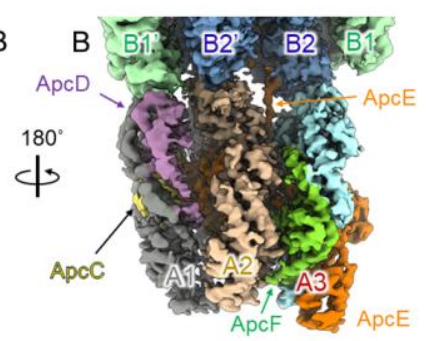

A

G

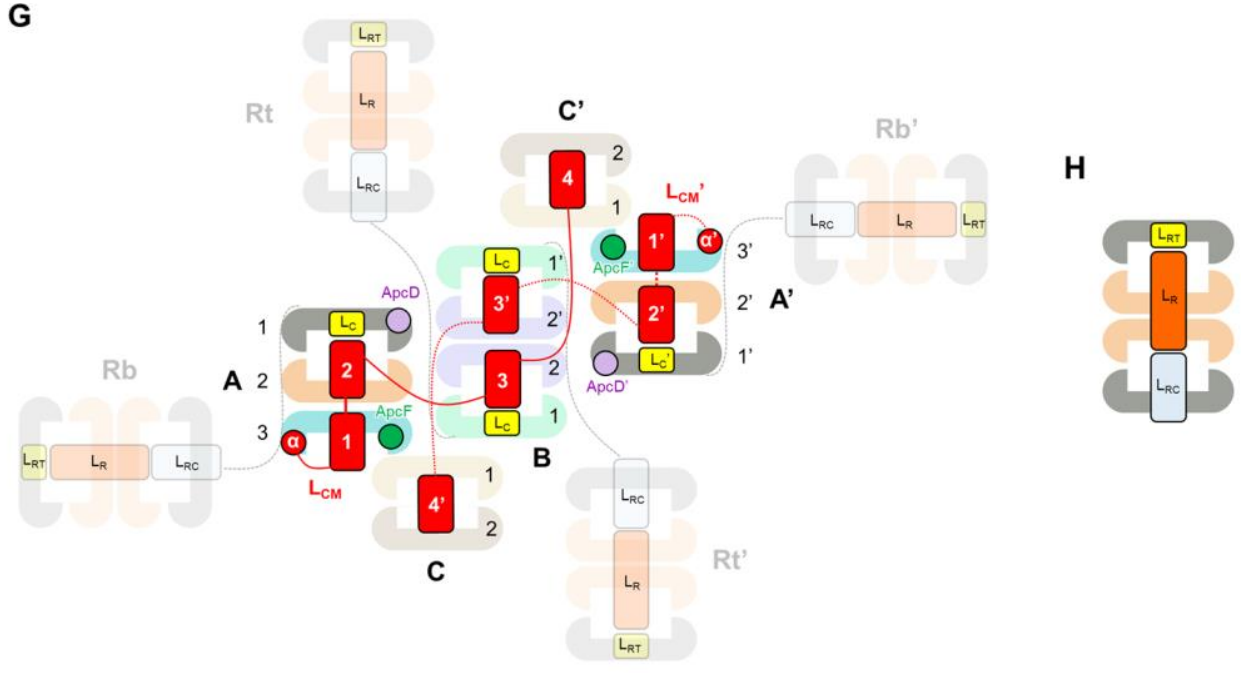

114 Fig. 1. Overall structure of the PBS core from T. vulcanus. (A-F) Cryo-EM density map of the PBS core from the front $(\mathrm{A}, \mathrm{C})$, side (B, E, and F), and bottom (D) views. (C) shows the

116 superposition of the cryo-EM density map and the refined PBS core model. (E) shows the

117 structure inside the rectangle in $(\mathrm{A})$, enlarged and rotated $90^{\circ} .(\mathrm{F})$ shows $(\mathrm{E})$ rotated $180^{\circ} .(\mathbf{G})$

118 Schematic model of the pentacylindrical APC core (including A (A'), B, and C (C') cylinders) 
119

120

121

122

123

124

125

126

127

128

129

130

131

132

133

134

135

136

137

138

139

and PC rods ( $\mathrm{Rb}, \mathrm{Rb}$ ', Rt, and $\mathrm{Rt}$ '). ApcE ( $\left.\mathrm{L}_{\mathrm{CM}}\right)$ containing $\alpha$, Reps $1-4$ and Arms $1-3$ is shown in red. PC rods that did not build the model are shown in translucent. The PC rod models were drawn referencing the cryo-EM map in this study and the PBS structure from Anabaena sp. PCC $7120^{5}$. (H) Schematic model of the PC rod. CpcC $\left(\mathrm{L}_{\mathrm{R}}\right), \mathrm{CpcD}\left(\mathrm{L}_{\mathrm{RT}}\right)$, and $\mathrm{CpcG}\left(\mathrm{L}_{\mathrm{RC}}\right)$ interact within the two PC hexamers.

The A cylinder is composed of the $\alpha$-subunits of phycobiliproteins (ApcA, ApcD, and $L_{C M}$ ), the $\beta$-subunits of phycobiliproteins (ApcB and ApcF), and ApcC (Lc). The A (A') cylinder consists of three APC trimers (A1, A2, and A3) with subunits of ApcD/ApcB and two ApcA/ApcB (A1); three ApcA/ApcB (A2); and ApcE/ApcB, ApcA/ApcF, and ApcA/ApcB (A3), respectively. Electrophoretic analysis shows that ApcD is present in prepared PBSs (Extended Data Fig. 2) ${ }^{17}$, but $\mathrm{ApcD}$ could not be identified in the cryo-EM density map due to the limited resolution. Then, we tentatively assigned $\mathrm{ApcD}$ to a subunit that could not be identified as ApcA (Extended data Fig. 6). The B cylinder consists of four APC trimers consisting of ApcA and ApcB (B1, B2, B1', and B2'). In addition, $\mathrm{L}_{\mathrm{C}}$ interacts with one side of the $\mathrm{A}$ and $\mathrm{C}$ cylinders, while it interacts with both sides of the B cylinder. The C cylinder consists of two APC trimers, ApcA and ApcB, which correspond to a half B cylinder.

$\mathrm{L}_{\mathrm{CM}}$ is a terminal emitter that is located at the membrane surface in the A cylinder and transfers energy to PSII. $\mathrm{L}_{\mathrm{CM}}$ is composed of $\alpha^{\mathrm{LCM}}$, showing a similar structure to ApcA, and has four repeated motifs named "repeats" (Rep1-Rep4) and "arms" (Arm1-3) that connect the motifs. Rep1 interacts with ApcF, two ApcAs ( $\alpha 2$ and $\alpha 3$ ), and one ApcB ( $\beta 1)$ in A1 (Fig. 2B). 

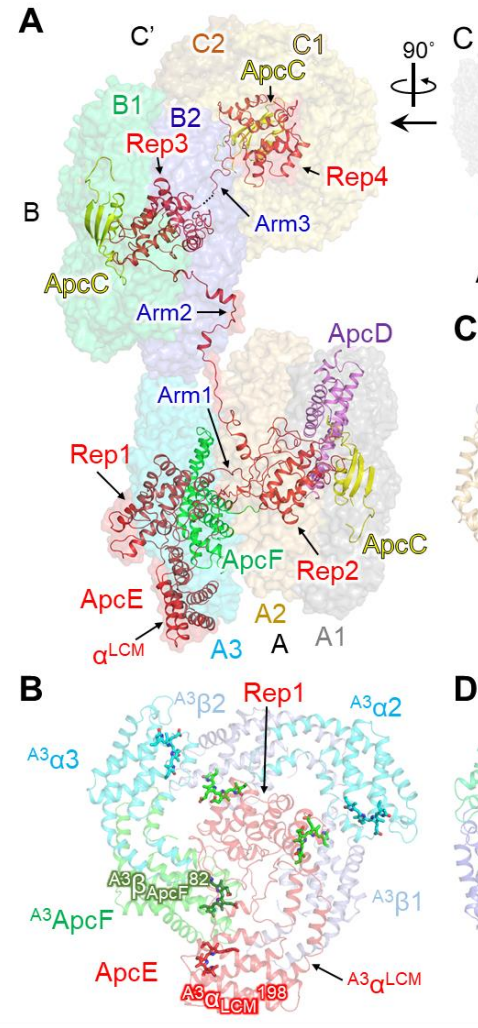

D

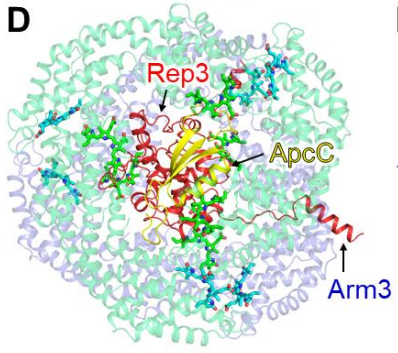

C' $180^{\circ} 80^{\circ} \quad$ C

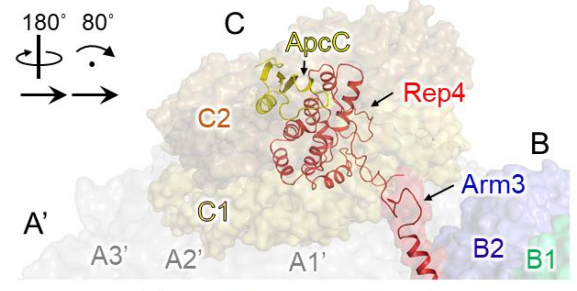

E

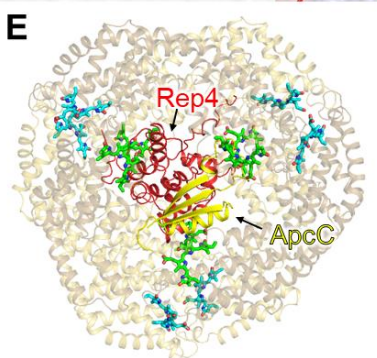

F

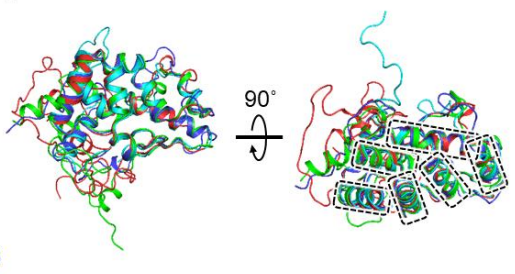
In the recently reported structures of red algae, ApcC interacts with Rep1 of $\mathrm{L}_{\mathrm{CM}}$, whereas ApcC

Fig. 2. Structures of A, B, and C cylinders, including terminal emitters and linker proteins.

(A) Arrangement of the terminal emitters ( $\alpha^{\mathrm{LCM}}$ of $\mathrm{L}_{\mathrm{CM}}, \mathrm{ApcD}$, and $\left.\mathrm{ApcF}\right)$ and linker proteins ( $\mathrm{L}_{\mathrm{CM}}$ and ApcCs). (B) Structures of APC trimer (A3) and Rep1 of $\mathrm{L}_{\mathrm{CM}}$ in A cylinder. (C) Structures of APC trimers A1 and A2, ApcC, and Rep2 of $\mathrm{L}_{\mathrm{CM}}$ in A cylinder. (D) Structures of APC trimers B1 and B2, ApcC, Rep3, and Arm3 of $\mathrm{L}_{\mathrm{CM}}$ in B cylinder. (E) Structure of APC trimers $\mathrm{C} 1$ and C2, ApcC, and Rep4 of $\mathrm{L}_{\mathrm{CM}}$ in C' cylinder. (F) Superposition of four Rep structures (Reps1-4). Dashed lines indicate the $\alpha$-helixes in the Rep regions. Rep1, green; Rep2, red; Rep3, blue; Rep4, cyan. and ApcD interact with Rep2 of the PBS core of T. vulcanus, indicating that the interaction 
153 between subunits in the core also differs between species. Rep3 of $\mathrm{L}_{\mathrm{CM}}$, together with ApcC,

154 contribute to the maintenance of the structure and function of the two APC trimers in the B

155

156

157

158

159

160

161

162

163

164

165

166

167

168

169

170

171

172

173

174

175

cylinder. Arm3 extends to Rep4, which serves as the linker domain of the C cylinder. Rep4

interacts with ApcC in the same way as Rep2 and Rep3, which means that each cylinder in the

PBS core contains one ApcC (Figs. 2C-E). An interesting feature of Reps $1-4$ in $\mathrm{L}_{\mathrm{CM}}$ is that the

structures of the $\alpha$-helix regions are similar, whereas the structures of the loop regions differ

(Fig. 2F). Sequence homology among Reps is not high (Extended Data Fig. 7), suggesting not

only that Reps 1-4 maintain the structure of each cylinder but also that distinct amino acid

residues in each Rep around PCB could contribute to efficient transfer of absorbed light energy

to the terminal emitters. Arm3, Rep4 of $\mathrm{L}_{\mathrm{CM}}$, and the $\mathrm{C}$ cylinder are not present in the PBS core

of red algae $\mathrm{e}^{13,14}$ or Synechocystis sp. PCC $6803^{4}$, whereas these components are present in about

half of cyanobacteria if the $\mathrm{L}_{\mathrm{CM}}$ domains are mapped to the phylogenetic tree of major

cyanobacterial species (Extended Data Figs. 8 and 9). Thus, common to many species of algae

including cyanobacteria, the C cylinder, Rep4, and Arm3, which maintain the C cylinder itself,

must play a key role in light-harvesting. $\mathrm{L}_{\mathrm{CM}}$ is one of the most important linker proteins for

energy transfer to PSII. The structure of T. vulcanus PBS described in this study provides a

starting point towards understanding component architecture and organizational networks for

general working mechanisms of PBSs in the broad algae group.

\section{Arrangement of chromatophores in the PBS core and energy transfer pathway}

The excitation energy is transferred to the terminal emitters $\left(\mathrm{L}_{\mathrm{CM}}, \mathrm{ApcD}\right.$, and $\left.\mathrm{ApcF}\right)$ via

chromophores in the B and C (C') cylinders, and the energy is eventually transferred to PSII and

PSI. T. vulcanus has PCB as its only chromophore, and it is the protein environment around each 
176 chromophore that must be intimately involved in the transfer of the excited energy to the

177 terminal emitters. Indeed, amino acid residues with polar/charged groups affect the absorption

178 energy of pigments ${ }^{33}$. In addition, an asparagine residue in the $\beta$ subunits is methylated (ligand

179 ID: MEN), and its mutation prevents the growth of cyanobacteria under high light conditions,

180 indicating its importance in energy transfer ${ }^{34}$.

181 Figure 3 shows each cylinder that constitutes the PBS core, the chromophores in the core, and

182 the distances between chromophores. In general, the excitation energy is transferred between

183 close pairs of chromophores, i.e., the donor (D) and acceptor (A) (Förster Resonance Energy

184 Transfer $)^{35,36}$. There are four main factors associated with excitation energy transfer rate: (i) the

185 distance between $\mathrm{D}$ and $\mathrm{A}$; (ii) the orientation factor, $\kappa^{2}$, which is a factor that describes the

186 relative orientation of the transition dipoles of D and A in space (Extended data Fig. 10); (iii) the

187 overlap integral between the fluorescence and absorption spectra of D and A, respectively; and

188 (iv) quantum yield of $\mathrm{D}$ in the absence of $\mathrm{A}$. For a freely rotating pigment $\mathrm{D}$ and $\mathrm{A}, \kappa^{2}$ has a

189 mean value of $2 / 3$, and the values of $\kappa^{2}$ the significant chromophores are approximately $1-3$

190 (Extended Data Table 5, Extended Data Figs. 10 and 11). This indicates that energy transfer is

191 likely to occur between their chromophores in the PBS core. The efficiency is inversely

192 proportional to the sixth power of the distance between D and A through dipole-dipole coupling;

193 thus, the shorter the distance between chromophores, the greater is the efficiency of the energy

194 transfer between them. Therefore, energy transfer efficiency between cylinders is strongly also

195 dependent on the distance between close chromophores. 

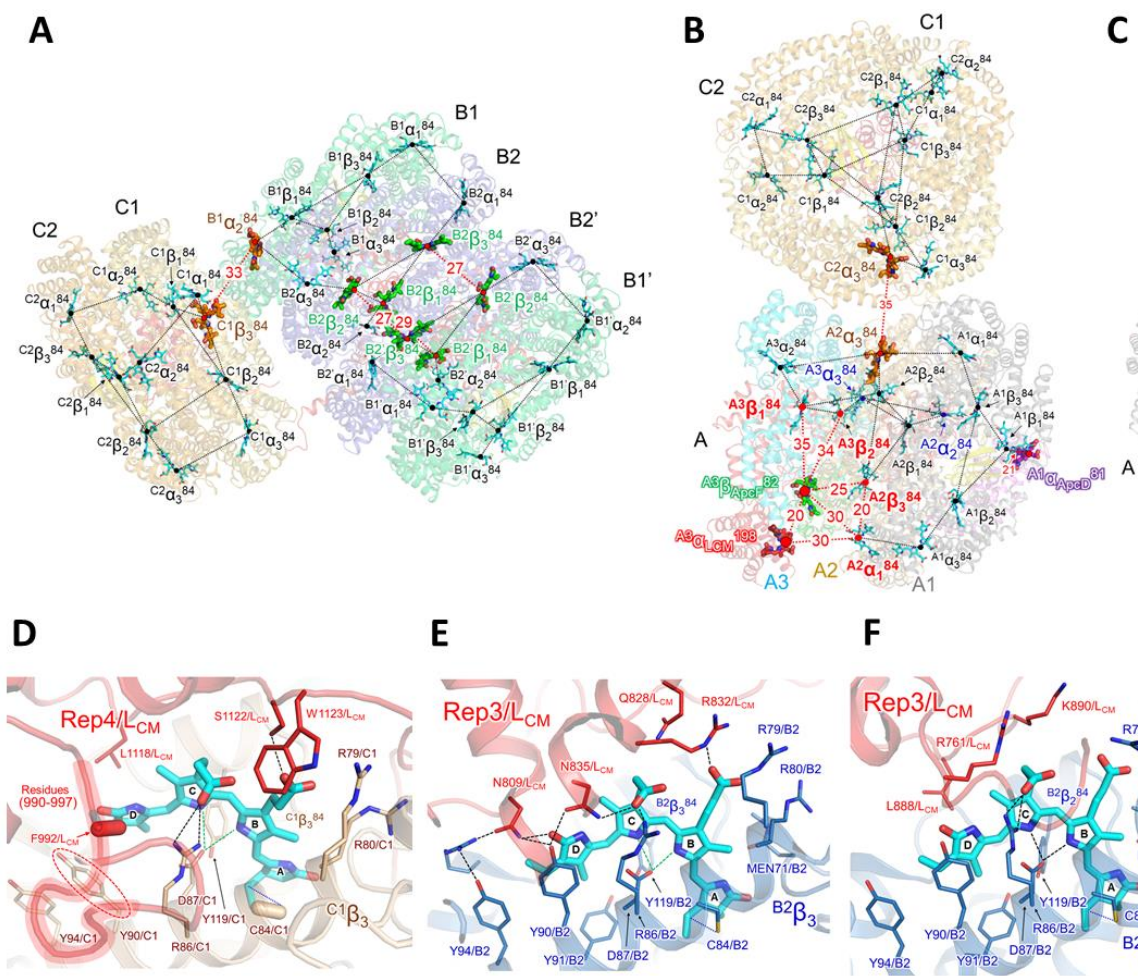

E

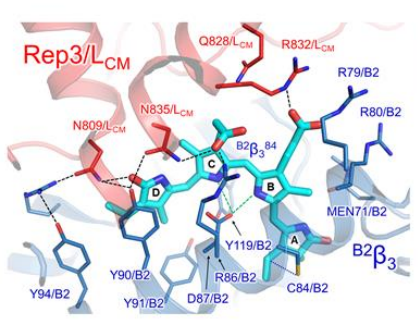

$\mathbf{F}$

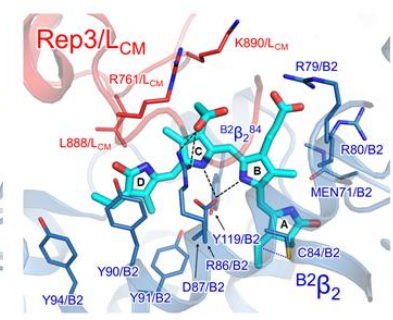

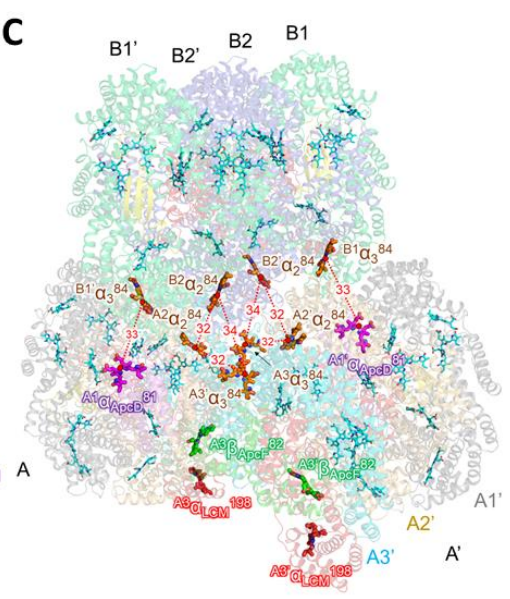

G

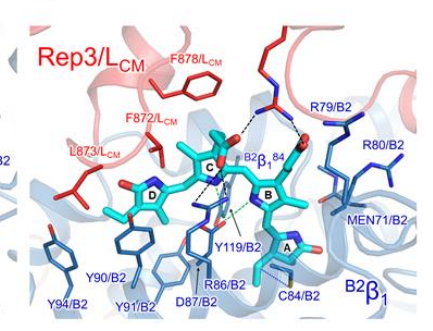

197

198

199

\section{Fig. 3. Arrangement of chromophores is the key to the energy transfer pathway in PBS}

core. (A) Possible energy transfer pathways in and between B and C (C') cylinders. (B) Possible energy transfer pathways in and between A and C cylinders. (C) Possible energy transfer pathways between A (A') and B cylinders. (D-G) The chromophores and the surrounding amino acid residues of $\mathrm{L}_{\mathrm{CM}}$. The numbers near the dotted lines indicate the distances $(\AA)$ between the PCB pairs. In (E-G), dashed lines (black and green) indicate hydrogen bonds, and dotted lines (blue) indicate covalent bonds between PCB and cysteine residue. In the amino acid residues (Phe992/L $\mathrm{L}_{\mathrm{CM}}$ and Cys84/C1) in (D), the directions of atoms from $\mathrm{C}_{\alpha}$ to $\mathrm{C}_{\beta}$ in the residues are indicated by the capsule-shaped objects.

The chromophores in the PBS core are numbered according to the nomenclature of those in red $\operatorname{alga}^{13,14}$, and their names are defined as follows: cylinder name (A, B, or C), subunit 
composition ( $\alpha$ or $\beta$ ), and the number of cysteine residues (84) to which the chromophore is bound (Extended Data Fig. 12). Again we reiterate that the previous red algal PBS structures do not contain $\mathrm{C}$ cylinders ${ }^{13,14}$. The chromophores involved in the energy transfer between or within each cylinder are shown in Fig. 3A-C. The energy transfer from the $\mathrm{C}\left(\mathrm{C}^{\prime}\right)$ cylinder to the $\mathrm{B}$ and

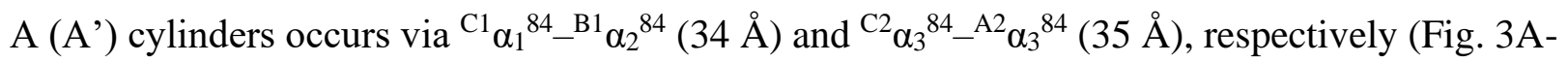
B). In the present structure, the D ring of PCB in many ApcBs forms $\pi-\pi$ interactions with tyrosine residues (Y90), but the D ring of ${ }^{\mathrm{C} 1} \beta_{3}{ }^{84}$ likely interacts with Phe992 within the loop region (residues 990-997) of Rep4 in the $\mathrm{L}_{\mathrm{CM}}$ (Fig. 3D). In addition, ${ }^{\mathrm{C} 1} \beta_{3}{ }^{84}$ interacts with S1122/LCM. These interactions probably contribute to energy transfer from the C (C') cylinder to the B cylinder. In the cyanobacterial PBS with bound PC rods (Rt, Rb, Rs1, and Rs 2$)^{5,17}$, the energy received by the $\mathrm{PC}$ rods is believed to be transferred to the $\mathrm{B}$ and $\mathrm{A}\left(\mathrm{A}^{\prime}\right)$ cylinders via the $\mathrm{C}\left(\mathrm{C}^{\prime}\right)$ cylinder, indicating that the $\mathrm{C}\left(\mathrm{C}^{\prime}\right)$ cylinder acts as an intermediary for energy transfer from the PC rods to the terminal emitters.

The B cylinder consists of four APC trimers, with two APC trimers (B2 and B2') interacting with each other. This indicates that energy transfer in the $\mathrm{B}$ cylinder is mediated by ${ }^{\mathrm{B} 2} \beta_{2}{ }^{84}-\mathrm{B}^{2} \beta_{1}{ }^{84}$ (27 $\AA$ ) ${ }^{\mathrm{B} 2} \beta_{1}{ }^{84}{ }^{\mathrm{B} 2}{ }^{\prime}{ }_{1}{ }^{84}(29 \AA)$, and ${ }^{\mathrm{B} 2} \beta_{3}{ }^{84}{ }^{\mathrm{B} 2}{ }^{\prime} \beta^{84}$ (27 $\left.\mathrm{\AA}\right)$ (Fig. 3A). Energy transfer from the B cylinder to the A (A') cylinder seems to involve ${ }^{\mathrm{B} 1} \alpha_{3}{ }^{84}-{ }^{\mathrm{A} 1} \alpha_{\mathrm{ApcD}}{ }^{81}(33 \AA)$ and ${ }^{\mathrm{B} 2} \alpha_{2}{ }^{84}-{ }^{\mathrm{A} 3^{\prime}} \alpha_{3}{ }^{84}$ (34 $\AA$ ) (Fig. 3C). In addition, there is an interaction between the A and A' cylinders, suggesting energy transfer occurs through ${ }^{\mathrm{A} 2} \alpha_{2}{ }^{84}{ }^{\mathrm{A} 3}{ }^{1} \alpha_{3}{ }^{84}$ (32 Å). These chromophores interact with Rep3 and Rep4 of $\mathrm{L}_{\mathrm{CM}}$, and the amino acid residue compositions of $\mathrm{L}_{\mathrm{CM}}$ differ around each of the four chromophores $\left({ }^{\mathrm{C} 1} \beta_{3}{ }^{84},{ }^{\mathrm{B} 2} \beta_{3}{ }^{84},{ }^{\mathrm{B} 2} \beta_{2}{ }^{84}\right.$, and ${ }^{\mathrm{B} 2} \beta_{1}{ }^{84}$ ) (Fig. 3D-G). Three chromophores $\left({ }^{\mathrm{B} 2} \beta_{3}{ }^{84},{ }^{\mathrm{B} 2} \beta_{2}{ }^{84}\right.$, and ${ }^{\mathrm{B} 2} \beta_{1}{ }^{84}$ ) interact with amino acid residues in $\mathrm{Rep}_{3} / \mathrm{L}_{\mathrm{CM}}$, significantly altering the peripheral structure of each chromophore. ${ }^{\mathrm{B} 2} \beta_{3}{ }^{84}$ forms hydrogen bonds with asparagine residues 
$232\left(\mathrm{~N} 809 / \mathrm{L}_{\mathrm{CM}}\right.$ and $\left.\mathrm{N} 835 / \mathrm{L}_{\mathrm{CM}}\right)$, and basic amino acids $\left(\mathrm{R} 762 / \mathrm{L}_{\mathrm{CM}}\right.$ and $\left.\mathrm{K} 890 / \mathrm{L}_{\mathrm{CM}}\right)$ are present near

$233{ }^{\mathrm{B} 2} \beta_{2}{ }^{84} \cdot{ }^{\mathrm{B} 2} \beta_{1}{ }^{84}$ interacts with a basic residue ( $\left.\mathrm{R} 730 / \mathrm{L}_{\mathrm{CM}}\right)$ and is surrounded by hydrophobic amino 234 acids $\left(\mathrm{L} 873 / \mathrm{L}_{\mathrm{CM}}, \mathrm{T} 872 / \mathrm{L}_{\mathrm{CM}}\right.$, and $\left.\mathrm{F} 878 / \mathrm{L}_{\mathrm{CM}}\right)$. This difference in the protein environment around 235 each chromophore may define their individual energy level.

\section{Excited energy transfer to terminal emitters}

238 Three PCBs in the A cylinder $\left({ }^{\mathrm{A} 2} \alpha_{3}{ }^{84},{ }^{\mathrm{A} 2} \alpha_{2}{ }^{84}\right.$, and $\left.{ }^{\mathrm{A} 3} \alpha_{3}{ }^{84}\right)$ are believed to be the key chromophores

239 that receive excitation energy from the B and C cylinders based on their spatial arrangement

240 (Fig. 3B), which then ultimately pass it to the two chromatophores $\left({ }^{\mathrm{A} 3} \beta_{\mathrm{ApcF}}{ }^{82}\right.$ and ${ }^{\mathrm{A} 3} \alpha_{\mathrm{LCM}}{ }^{198}$ ) in

241 the terminal emitters. In this case, the excitation energy is most likely transferred via the four

242 chromophores $\left({ }^{\mathrm{A} 2} \alpha_{1}{ }^{84},{ }^{\mathrm{A} 2} \beta_{3}{ }^{84},{ }^{\mathrm{A} 3} \beta_{1}{ }^{84}\right.$, and ${ }^{\mathrm{A} 3} \beta_{2}{ }^{84}$ ) close to these two chromophores. Three of the

243 four chromophores interact with Rep1 or Rep2, in a characteristic structure (Fig. 4A-C). The $\kappa^{2}$

244 value and the distance between chromophores also suggest that the energy transfer efficiency of

$245{ }^{\mathrm{A} 3} \alpha_{\mathrm{LCM}}{ }^{198}{ }^{\mathrm{A} 3} \beta_{\mathrm{ApcF}}{ }^{82}$ is highest among all the identified pairs (Extended Data Table 5). 


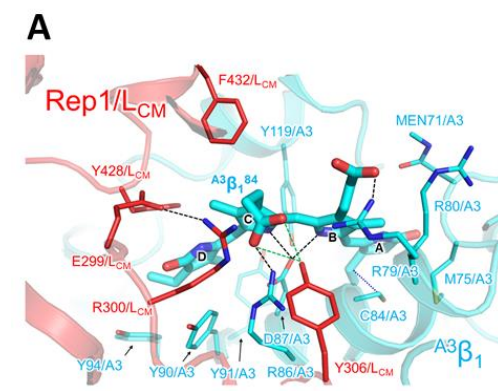

D

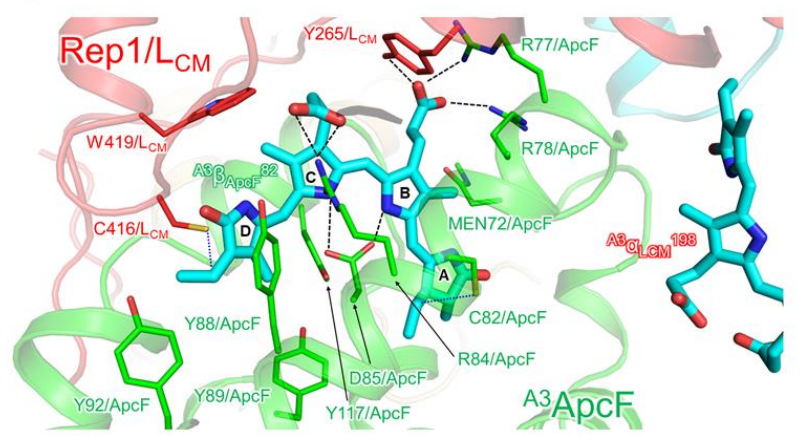

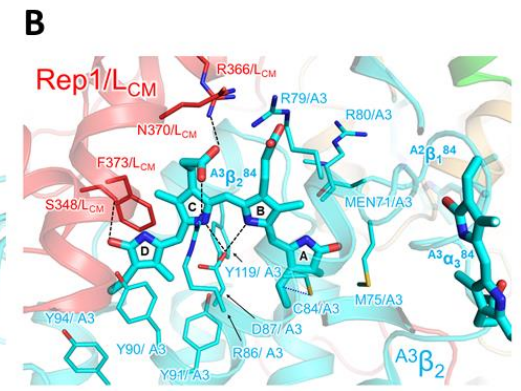

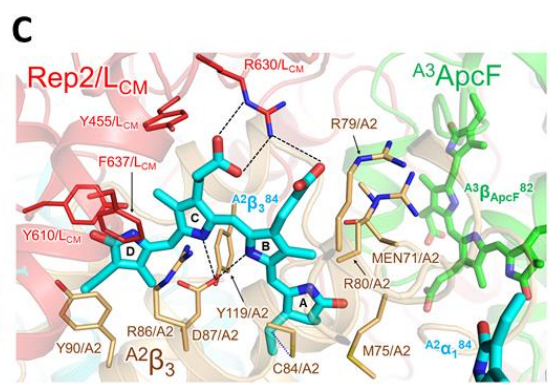

E

\section{Fig. 4. Chromophores and their surrounding structures in terminal emitters (ApcF and}

LCM). (A-B) Chromophores in the APC trimer A3 and their interaction with Rep1 of $\mathrm{L}_{\mathrm{CM} .}$ (C)

Chromophore in the APC trimer A2 and its interaction with Rep2 of $\mathrm{L}_{\mathrm{CM}}$. (D) Chromophore of ApcF and its interaction with Rep1 of $\mathrm{L}_{\mathrm{CM}}$. (E) Chromophore of $\mathrm{L}_{\mathrm{CM}}$ and its interaction with ApcF. The dashed lines (black) indicate hydrogen bonds. The dotted lines (blue) indicate covalent bonds between PCB and cysteine residue. The directions of atoms from $\mathrm{C} \alpha$ to $\mathrm{C} \beta$ in the residues are indicated by the capsule-shaped objects.

${ }^{\mathrm{A} 3} \beta_{1}{ }^{84}$ interacts with $\mathrm{Y} 306 / \mathrm{L}_{\mathrm{CM}}$ and is surrounded by aromatic amino acids (Y428/L $\mathrm{CM}$ and F432/LCM). ${ }^{\mathrm{A} 3} \beta_{2}{ }^{84}$ interacts with $\mathrm{S} 348 / \mathrm{L}_{\mathrm{CM}}$ and $\mathrm{R} 366 / \mathrm{L}_{\mathrm{CM}}$, and $\mathrm{F} 373 / \mathrm{L}_{\mathrm{CM}}$ is close to the $\mathrm{D}$ ring of ${ }^{\mathrm{A} 3} \beta_{2}{ }^{84}$. In addition, ${ }^{\mathrm{A} 2} \beta_{3}{ }^{84}$ interacts with $\mathrm{R} 630 / \mathrm{L}_{\mathrm{CM}}$ and is surrounded by three aromatic amino acids (Y455/L $\mathrm{CM}, \mathrm{Y} 610 / \mathrm{L}_{\mathrm{CM}}$, and $\left.\mathrm{F} 637 / \mathrm{L}_{\mathrm{CM}}\right)$, with $\mathrm{F} 637 / \mathrm{L}_{\mathrm{CM}}$ close to the D ring of ${ }^{\mathrm{A} 2} \beta_{3}{ }^{84}$. It was noted that, in the structure of red algal PBSs, aromatic amino acids are abundant in the vicinity 
of the chromophores and it was proposed that they regulate the energy state of each chromophore to achieve unidirectional energy transfer ${ }^{13,14}$. The aromatic amino acids in the linker proteins of T. vulcanus PBS may function similarly, suggesting that the protein environment around the three chromophores is optimized for efficient transfer of excitation energy to the two chromophores.

The chromophore ${ }^{\mathrm{A} 3} \beta_{\mathrm{ApcF}}{ }^{82}$ in $\mathrm{ApcF}$ binds $\mathrm{C} 82 / \mathrm{ApcF}$ as well as $\mathrm{C} 416 / \mathrm{L}_{\mathrm{CM}}$ and interacts with four amino acid residues (Y265/ $\mathrm{L}_{\mathrm{CM}}, \mathrm{R} 84 / \mathrm{ApcF}, \mathrm{R} 77 / \mathrm{ApcF}$, and R78/ApcF). In the absence of both ApcF and ApcD, the PBS is unable to transfer energy to either PSII or PSI, and thus the major route of energy transfer to PSII and PSI appears to be through ApcF rather than ApcD ${ }^{37}$. Thus, the protein environment around ${ }^{\mathrm{A} 3} \beta_{\mathrm{ApcF}}{ }^{82}$ in ApcF seems key for transferring the unidirectional excitation energy to PSII and PSI. ApcD is a terminal emitter that transfers energy to $\mathrm{PSI}^{37}$, and the present study suggests that ${ }^{\mathrm{A} 1} \beta_{1}{ }^{84}$ and ${ }^{\mathrm{B} 1} \alpha_{3}{ }^{84}$ are involved in the energy transfer pathway to ${ }^{\mathrm{A} 1} \alpha_{\mathrm{ApcD}}{ }^{81}$.

We were not able to identify the side chains of three amino acid residues (W166/Lcm, D163/LcM, and $\mathrm{K} 159 / \mathrm{L}_{\mathrm{CM}}$ ), as the cryo-EM density map around ${ }^{\mathrm{A} 3} \alpha_{\mathrm{LCM}}{ }^{198}$ was somewhat disordered (Fig. could form a $\pi-\pi$ interaction with $\mathrm{W} 166 / \mathrm{L}_{\mathrm{CM}}$, as the D-ring of PCB and tyrosine residues in

277 ApcA commonly form a $\pi-\pi$ interaction. This feature is probably an important factor for energy transfer from ${ }^{\mathrm{A} 3} \alpha_{\mathrm{LCM}}{ }^{198}$ to PSII. Further details with regard to ${ }^{\mathrm{A} 3} \alpha_{\mathrm{LCM}}{ }^{198}$ and key interactions with

279 surrounding structures will need a higher-resolution structure of the PBS and/or a

280 supercomplex structure interacting with PBS and PSII.

\section{Structure of PC rod and arrangement of chromatophores}



with each other (Fig. 5A) and three linker proteins inside the rod. CpcC, CpcD, CpcG1, CpcG2, and CpcG4 have been identified as linker proteins of the PC in T. vulcanus PBSs (Extended Data Fig. 2) ${ }^{17}$, and we could assign $\mathrm{CpcC}, \mathrm{CpcD}$, and $\mathrm{CpcG} 2$ in the cryo-EM map of the PC rod (Fig.

13), CpcG2 assignment in this study is tentative.

A

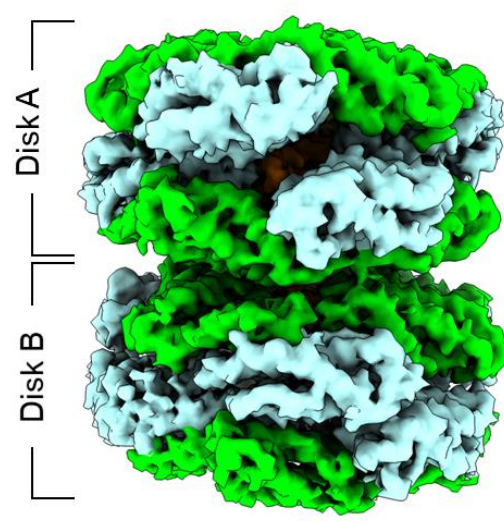

D

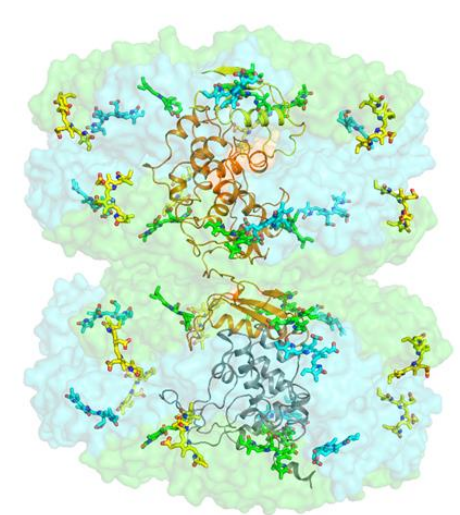

B

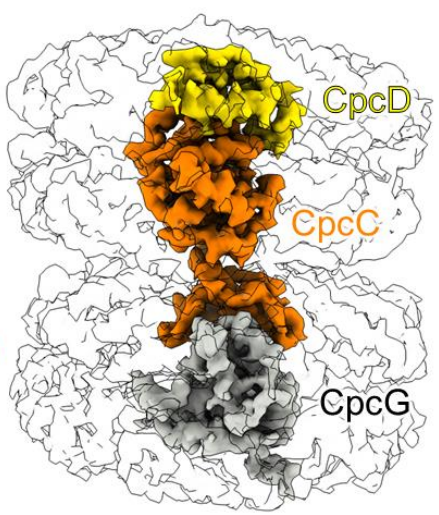

E

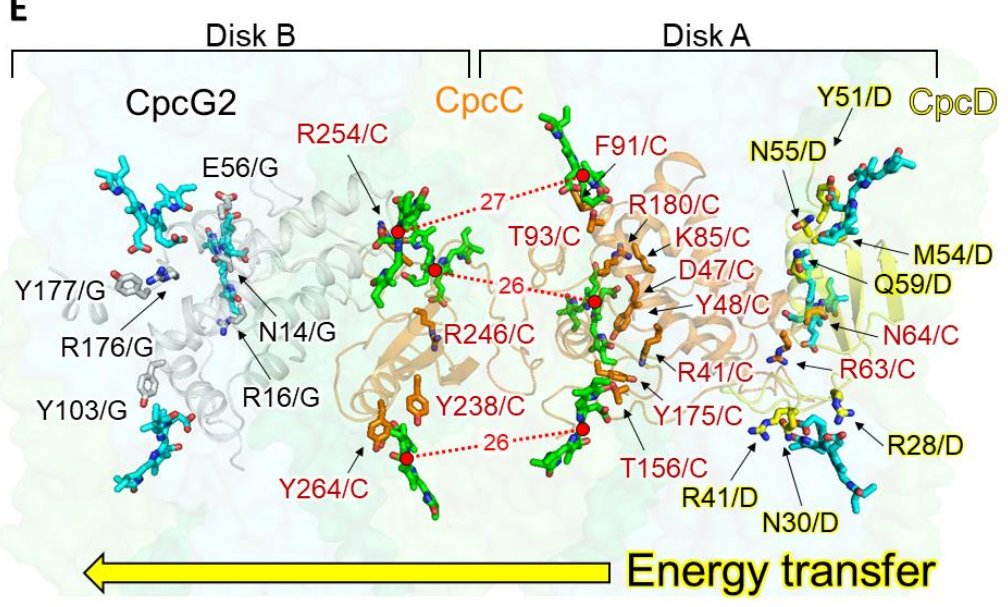

Fig. 5. Structure of PC rod, including linker proteins, and its energy transfer pathway. (A)

cryo-EM density map of the PC rod (Disk A and Disk B). (B) Arrangement of the linker proteins $(\mathrm{CpcC}, \mathrm{CpcD}$, and $\mathrm{CpcG})$ in the PC rod. (C) Structure of the PC rod in (B) rotated $90^{\circ}$. (D) Arrangement of chromophores in the PC rod. $\alpha^{84}, \beta^{84}$, and $\beta^{155}$ are colored cyan, green, and 
294 yellow, respectively. (E) Arrangement of $\beta^{84}$ s in a PC rod interacting with linker proteins. $\beta^{84}$ s at

295 the boundary between Disk A and Disk B are colored green, and these chromophores are

296 involved in the energy transfer between Disk A and Disk B. The amino acid residues of the

297 linker proteins near $\beta^{84} \mathrm{~s}$ are indicated in $(\mathrm{E})$.

The PC rods dissociated from the PBS should contain different CpcG subunits. In the Anabaena PBS, when three CpcG encoding genes ( $C p c G 1, C p c G 2$, and $C p c G 4)$ were deleted, the PC rods could not bind to the PBS core ${ }^{5}$. When one of the $\mathrm{CpcG}$ encoding genes was deleted, the PC rods could interact with the PBS core but not in the same manner as the wild-type PBS. This suggests that the composition of the $\mathrm{CpcG}$ protein is different in each $\mathrm{PC} \operatorname{rod}^{5}$. The cryo-EM density map resolves the linker proteins extending from the PC rods interacting with the PBS core, with a resolution enough to see (Fig. 1, Extended Data Fig. 14). The C-terminal region of the CpcG protein in $\mathrm{Rt}\left(\mathrm{Rt}^{\prime}\right)$ and $\mathrm{Rb}\left(\mathrm{Rb}^{\prime}\right)$ mainly interacts with the peripheral region of the $\mathrm{B}$ cylinder and the peripheral region of the A (A') cylinder, respectively. The C-terminal region of $\mathrm{CpcG}$ interacts weakly with the cylinders of the PBS core and also supports a rod.

The PC structures obtained by X-ray crystal structure analysis do not include any linker proteins, and the interaction between the PC monomers in the oligomer is symmetric ${ }^{9}$. In contrast, the

311 interaction between PCs and linker proteins in the PC rod is asymmetric (Fig. 5A and D), which

312 is induced by the interactions between $\mathrm{CpcC}, \mathrm{CpcD}$, and $\mathrm{CpcG} 2$ in the rod. Consequently, the

313 arrangement of chromophores, especially at the boundary between Disks A and B in the PC rod, 314 is different from that inferred from the crystal packing of the PC structure (Fig. 5D, Extended

315 Data Fig. 15). CpcD ( $\left.\mathrm{L}_{R}\right)$, a small rod cap linker domain, is the outermost linker protein of the 316 intact PBS, and the excitation energy is transferred from Disk A to Disk B in the PC rod via the 
317 chromophores $(\beta 84 \mathrm{~s})$ interacting with $\mathrm{CpcC}$ (distances between chromophores are 26-27 $\mathrm{A}$ )

318 (Fig. 5E). A significant difference is noted in the chromophore arrangement in the cryo-EM map,

319 especially in Disk B, although the observed distances were almost the same as those predicted

320 from the crystal structure analysis. This difference is apparently caused by interaction of linker

321 proteins (especially $\mathrm{CpcC}$ and $\mathrm{CpcG} 2$ ) (Extended Data Fig. 15). In previous studies on PC using

322 spectroscopy, $\beta 84$ in a PC rod interacting with linker proteins was proposed to be involved in the

323 energy transfer between PC hexamers ${ }^{38-40}$. Therefore, six $\beta 84$ s (Fig. 5E) at the boundary between

324 Disks A and B are probably involved in the energy transfer between the disks. The characteristic

325 amino acid residues around the chromophores in the linker proteins and the interactions between

326 these residues and the chromophores are likely crucial for unidirectional energy transfer in the

327 PC rod.

\section{References}

1. Sidler, W. A. Phycobilisome and Phycobiliprotein Structures (Advanced Photosynthesis

2. Li, Y. et al. Characterization of red-shifted phycobilisomes isolated from the chlorophyll f-

3. Scheer, H. \& Zhao, K. H. Biliprotein maturation: the chromophore attachment. Mol. containing cyanobacterium Halomicronema hongdechloris. Biochim. Biophys. Actaseries, Springer, Dordrecht), pp. 139-216.

$$
\text { Bioenerg. 1857, 107-114 (2016). https://doi.org/10.1016/j.bbabio.2015.10.009 }
$$


5. Chang, L. et al. Structural organization of an intact phycobilisome and its association with photosystem II. Cell Res. 25, 726-737 (2015).

6. Arteni, A. A., Liu, L. N., Aartsma, T. J., Zhang, Y. Z., Zhou, B. C. \& Boekema, E. J. Structure and organization of phycobilisomes on membranes of the red alga Porphyridium cruentum. Photosynth. Res. 95, 169-174 (2008).

7. Gantt, E. \& Lipschultz, C. A. Structure and phycobiliprotein composition of phycobilisomes from Griffithsia pacifica (Rhodophyceae). J. Phycol. 16, 394-398 (1980).

8. Watanabe, M. et al. Attachment of phycobilisomes in an antenna-photosystem I supercomplex of cyanobacteria. Proc. Natl. Acad. Sci. U. S. A. 111: 2512-2517 (2014).

9. Hirose, Y.S. et al. Diverse chromatic acclimation processes regulating phycoerythrocyanin and rod-shaped phycobilisome in cyanobacteria. Mol. Plant. 12, 715-725 (2019).

10. Gerard Guglielmi, D. A. B. \& Cohen-Bazire, G. The structure of Gloeobacter violaceus and its phycobiliosomes. Arch. Microbiol. 129, 181-189 (1981).

11. Cohen-Bazire, G. \& Bryant, D. A. Phycobilisomes: composition and structure. In: Carr NG, Whitton BA (eds) The Biology of Cyanobacteria. Blackwell, Oxford London, p 143-190 (1982).

12. Collier, J. L. \& Grossman, A. R. A small polypeptide triggers complete degradation of lightharvesting phycobiliproteins in nutrient-deprived cyanobacteria, EMBO J., 13, 1039-1047 (1994).

13. Zhang, J. et al. Structure of phycobilisome from the red alga Griffithsia pacifica. Nature. 551, 57-63 (2017). 
14. Ma, J., You, X., Sun, S., Wang, X., Qin, S. \& Sui, S. F. Structural basis of energy transfer in Porphyridium purpureum phycobilisome. Nature. 579, 146-151 (2020).

15. Adir, N., Bar-Zvi, S. \& Harris, D. The amazing phycobilisome. Biochim. Biophys. Acta Bioenerg. 1861, 148047 (2020).

16. Zeng, et al., Structural insight into the mechanism of energy transfer in cyanobacterial phycobilisomes. Nature Commun. 12, 5497 (2021)

17. Kawakami, K. et al. Structural implications for a phycobilisome complex from the thermophilic cyanobacterium Thermosynechococcus vulcanus. Biochim. Biophys. Acta Bioenerg. 1862, 148458 (2021).

18. Liu, H., Zhang, M. M., Weisz, D. A., Cheng, M., Pakrasi, H. B. \& Blankenship, R. E. Structure of cyanobacterial phycobilisome core revealed by structural modeling and chemical cross-linking. Sci. Adv. 7, 1-11 (2021).

19. Zhao, K. H. et al. Phycobilin:cystein-84 biliprotein lyase, a near-universal lyase for cysteine84-binding sites in cyanobacterial phycobiliproteins. Proc. Natl. Acad. Sci. U. S. A. 104, 14300-14305 (2007).

20. Hirota, Y., Serikawa, H., Kawakami, K., Ueno, M., Kamiya, N. \& Kosumi, D. Ultrafast energy transfer dynamics of phycobilisome from Thermosynechococcus vulcanus, as revealed by ps fluorescence and fs pump-probe spectroscopies. Photosynth. Res. (2021), doi:10.1007/s11120-021-00844-0.

21. McGregor, A., Klartag, M., David, L. \& Adir, N. Allophycocyanin Trimer Stability and Functionality Are Primarily Due to Polar Enhanced Hydrophobicity of the Phycocyanobilin Binding Pocket. J. Mol. Biol. 384, 406-421 (2008). 
22. David, L., Marx, A. \& Adir, N. High-resolution crystal structures of trimeric and rod phycocyanin. J. Mol. Biol. 405, 201-213 (2011).

23. Adir, N., Dobrovetsky, Y. \& Lerner, N. Structure of c-phycocyanin from the thermophilic cyanobacterium Synechococcus vulcanus at $2.5 \AA$ : structural implications for thermal stability in phycobiliosme assembly. J. Mol. Biol., 71-81 (2001).

24. Adir, N. \& Lerner, N. The crystal structure of a novel unmethylated form of c-phycocyanin, a possible connector between cores and rods in phycobilisomes. J. Biol. Chem. 278, 25926$25932(2003)$

25. Murray, J. W., Maghlaoui, K. \& Barber, J. The structure of allophycocyanin from Thermosynechococcus elongatus at $3.5 \AA$ resolution. Acta Crystallogr. Sect. F. Struct. Biol. Cryst. Commun. 63, 998-1002 (2007).

26. Marx, A. \& Adir, N. Allophycocyanin and phycocyanin crystal structures reveal facets of phycobilisome assembly. Biochim. Biophys. Acta Bioenerg. 1827, 311-318 (2013).

27. David, L., Prado, M., Arteni, A. A., Elmlund, D. A., Blankenship, R. E. \& Adir, N. Structural studies show energy transfer within stabilized phycobilisomes independent of the mode of rod-core assembly. Biochim. Biophys. Acta Bioenerg. 1837, 385-395 (2014).

28. Kosourov, S., Murukesan, G., Seibert, M. \& Allahverdiyeva, Y. Evaluation of light energy to $\mathrm{H} 2$ energy conversion efficiency in thin films of cyanobacteria and green alga under photoautotrophic conditions. Algal Res. 28, 253-263 (2017).

29. Santos-Merino, M., Singh, A. K. \& Ducat, D. C. New applications of synthetic biology tools for cyanobacterial metabolic engineering. Front. Bioeng. Biotechnol. 7, 1-24 (2019). 
30. Kim, M. J., Lee, S., Moon, C. K., Kim, J. J., Youn, J. R. \& Song, Y. S. A Broadband Multiplex Living Solar Cell. Nano Lett. 20, 4286-4291 (2020).

31. Kastner, B. et al. GraFix: sample preparation for single-particle electron cryomicroscopy. Nat. Methods. 5, 53-55 (2008).

32. Pintilie, G., Zhang, K., Su, Z., Li, S., Schmid, M. F. \& Chiu, W. Measurement of atom resolvability in cryo-EM maps with Q-scores. Nat. Methods. 17, 328-334 (2020).

33. K. Saito, K. Mitsuhashi, H. Ishikita, Dependence of the chlorophyll wavelength on the orientation of a charged group: Why does the accessory chlorophyll have a low site energy in photosystem II? J. Photochem. Photobiol. A Chem. 402, 112799 (2020).

34. Miller, C. A. et al. Biogenesis of phycobiliproteins: III. CpcM is the asparagine methyltransferase for phycobiliprotein $\beta$-subunits in cyanobacteria. J. Biol. Chem. 283, 19293-19300 (2008).

35. Förster, T. Zwischenmolecculare energiewanderung und fluoreszen (1948), pp. 55-75.

36. Lubert Stryer, R. P. H. Energy transfer: a spectroscopic ruler. Proc. Natl Acad. Sci. USA. 58, 719-726 (1967).

37. Ashby, M. K. \& Mullineaux, C. W. The role of ApcD and ApcF in energy transfer from phycobilisomes to PS I and PS II in a cyanobacterium. Photosynth. Res. 61, 169-179 (1999).

38. Mimuro, M. Studies on excitation energy flow in the photosynthetic pigment system; structure and energy transfer mechanism. Bot. Mag. Tokyo. 103, 233-253 (1990).

39. Zhang, J., Zhao, F., Zheng, X. \& Wang, H. Direct measurement of excitation transfer dynamics between two trimers in C-phycocyanin hexamer from cyanobacterium Anabaena variabilis. Chem. Phys. Lett. 304, 357-364 (1999). 
40. Niedzwiedzki, D. M., Bar-Zvi, S., Blankenship, R. E. \& Adir, N. Mapping the excitation energy migration pathways in phycobilisomes from the cyanobacterium Acaryochloris marina. Biochim. Biophys. Acta Bioenerg. 1860, 286-296 (2019).

\section{Methods}

\section{Preparation of intact PBSs, PBS cores, and PC rods. Cells of the thermophilic cyanobacteria} Thermosynechococcus vulcanus NIES-2134 were cultured in a phosphate medium ${ }^{40,41}$. Intact PBSs and PBS cores were prepared according to the protocols described by Kawakami et al. ${ }^{17}$. Thylakoid membranes, PSI, and PSII were prepared according to the protocols described by Kawakami et al. ${ }^{40,41}$. For PC rods, the intact PBSs were mounted on a holey carbon film-coated copper grid (see "Sample preparation and data collection for cryo-EM" for a description of pre-treatment of the grid), and then a small amount of buffer containing $10 \mathrm{mM}$ phosphate buffer was quickly added to reduce the phosphate concentration in the sample to less than $0.5 \mathrm{M}$. This resulted in the dissociation of the PC rods from the intact PBSs.

Sample preparation and data collection for cryo-EM. To clarify the structure of the PBS core and PC rod, grids for cryo-EM were prepared in the following manner. A holey carbon filmcoated copper grid (Quantifoil R1.2/1.3 Cu 200 mesh, Microtools, GmbH, Berlin, Germany) that had been pretreated by Au sputtering was glow-discharged for $10 \mathrm{~s}$ using an ion coater (JEC3000FC, JEOL, Tokyo, Japan). For the PBS core, $3.0 \mu \mathrm{L}$ of the PBS core was applied to the grid and blotted with filter paper for $4 \mathrm{~s}$, then immediately plunge-frozen in cooled ethane using a FEI 
Vitrobot Mark IV (Thermo Fisher Scientific, Waltham, MA, USA) under 100\% humidity at $\mu \mathrm{L}$ of $10 \mathrm{mM}$ phosphate buffer on the grid. Then, the grid was blotted and manually plungefrozen in cooled ethane using a homemade plunger. The grids were then introduced into a CRYO ARM 300 electron microscope (JEOL) equipped with a cold-field emission gun and an incolumn energy filter with a slit width of $20 \mathrm{eV}$. Dose-fractionated images were recorded using a images were corrected using a JEOL automatic data acquisition system ${ }^{43}$ with a nominal magnification of $40,000 \times$, which corresponded to a pixel size of $1.24 \AA$. Nominal defocus ranges and dose rates for PBS core and PC rod were -0.5 to $-1.5 \mu \mathrm{m}$ and $84.1 \mathrm{e}^{-} \AA^{-2}$ with 50 frames, and -0.5 to $-1.5 \mu \mathrm{m}$ and $50.5 \mathrm{e}^{-} \AA^{-2}$ with 30 frames, respectively. In total, we collected 4,600 and 2,865 movies for the PBS core and PC rod, respectively.

Data processing. The collected movie stacks of the PBS core were divided into eight optics groups to correct changes in the beam tilt over time. Drift correction and dose-weighted frame summing were performed using MotionCor2-1.3.2 $2^{44}$, and contrast transfer function (CTF) parameters were estimated using CTFFIND4-1.10 45 . The images were selected for further data 466 processing based on the Thon ring patterns. The PBS core particles were manually picked and 467 subjected to reference-free, two-dimensional (2D) classification by RELION-3.1.0 ${ }^{46}$ to create 468 reference images for automatic particle picking. In all, 128,676 particles were picked 469 automatically and extracted with a pixel size of $2.48 \AA$. Good averaged classes containing 45,427 470 particles after 2D classification were subjected to ab initio three-dimensional (3D) reconstruction 471 in cryoSPARC-2.12.0 ${ }^{47}$. Following the 3D classification in RELION, a well-aligned 3D class 
472 containing 25,532 particles was extracted with a pixel size of $1.24 \AA$ and used for further $3 \mathrm{D}$

473 refinement with two-fold symmetry enforcement. Post-processing in RELION yielded a 4.75- $\AA$

474 resolution map based on the gold standard FSC. Finally, the resolution reached $3.71 \AA$ after two

475 rounds of Bayesian polishing and CTF refinement.

For PC12mer, all movie stacks were processed using MotionCor2-1.2.1 and Gctf-1.06 ${ }^{48}$

477 for drift correction, frame summation, and CTF parameter estimation. The 812,327 particles

478 were picked by convolutional neural network picking using EMAN-2.3.1 ${ }^{49}$ and binned to a pixel

479 size of $4.96 \AA$ A during extraction using RELION-3.0. After two rounds of 2D classification,

480309,291 particles were selected and subjected to ab initio model construction using cisTEM-

1.0.0 beta $^{50}$. Good particles were automatically picked again with a $3 \mathrm{D}$ reference using the

EMAN-2.3.1. In all, 159,537 particles with a pixel size of $1.86 \AA$ were selected from 1,022,349 picked particles based on 2D and 3D classifications. 3D refinement using RELION-3.0 was improved the resolution to $4.19 \AA$ with 111,054 particles without any symmetry enforcement. For further details, see Extended Data Figs. 4 and 5, and Extended Data Table 1. vulcanus were built using reference models of PC and APC (PDB codes: 3O18, 3DBJ) and homology modeling was performed on the SWISS-MODEL server

491 (https://swissmodel.expasy.org/) by referring to the structures of the linker proteins (PDB codes:

$4925 \mathrm{Y} 6 \mathrm{P}$ and $6 \mathrm{KGX})$. The obtained models were fitted with the cryo-EM density maps using the "fit 493 in map" program in UCSF Chimera (version 1.13), and initial models of the PBS core and PC 494 rod were created. The initial models were modified manually using COOT to fit the cryo-EM 
495 density map and refined using Phenix (version 1.19.2). Subsequently, each subunit and its

496 interacting subunits in the PBS core and PC rod were grouped and refined against the cryo-EM

497 density map using REFMAC5 (version 5.8.0267) in CCP-EM. Finally, the grouped models were

498 merged into one, and the overall structures (PBS core and PC rod) were refined using Phenix.

499 The refinement statistics of the refined models were obtained using the comprehensive validation

500 program in Phenix. The restraints needed for the ligands in the PBS core and PC rod from $T$.

501 vulcanus were generated by electronic Ligand Bond Builder and Optimization Workbench

$502(\mathrm{eLBOW})^{51}$. The restraint information for a methylated Asn (ligand ID: MEN) and

503 phycocyanobilin (ligand ID: CYC) was obtained from the model of MEN identified in a high-

504 resolution crystal structure (PDB code: 3O18). Comprehensive validation (in Phenix), Q-score ${ }^{32}$

505 and FSC-Q ${ }^{52}$ were used to validate the refined structural models (PBS core and PC rod).

507 Estimation of the orientation factor between chromophores in the PBS core. Based on the

508 arrangement of the PCBs and their orientation in the constructed PBS core, an approximate

509 orientation factor, $\kappa^{2}$, was estimated (Extended Data Table 5). These estimates were made

510 especially for the sites likely to be involved in the energy transfer between cylinders in the PBS

511 core and for the terminal emitters. Excitation energy transfer (EET) rate $\left(k_{\mathrm{EET}}\right)$ is given by the

512 following equation (1), where $V$ and $\Theta$ are the electronic coupling factor and the overlap integral, 513 respectively. 
517 As shown in fig. S10 (A), the transition dipole moment of donor (D) and acceptor (A) are $\boldsymbol{\mu}_{\mathbf{D}}$ and $518 \boldsymbol{\mu}_{\mathrm{A}}$, respectively. $\mathbf{r}$ is the intermolecular center-to-center distance between $\mathrm{D}$ and A. $V$ is written 519 by the approximation equation as shown in the following equations $(2,3)$, and the orientation 520 factor $\left(\kappa^{2}\right)$ can be estimated by equation (4).

$$
V \approx\left[\frac{\vec{\mu}_{\mathrm{D}} \cdot \vec{\mu}_{\mathrm{A}}}{r^{3}}-\frac{3\left(\vec{r} \cdot \vec{\mu}_{\mathrm{D}}\right)\left(\vec{r}_{\vec{\mu}}\right)}{r^{5}}\right]
$$

$$
\approx\left[\frac{\mu_{\mathrm{D}} \mu_{\mathrm{A}} \cos \theta_{\mathrm{T}}}{r^{3}}-\frac{3\left(\mu_{\mathrm{D}} r \cos \theta_{\mathrm{D}}\right)\left(\mu_{\mathrm{A}} r \cos \theta_{\mathrm{A}}\right)}{r^{5}}\right]=\frac{\mu_{\mathrm{D}} \mu_{\mathrm{A}}\left(\cos \theta_{\mathrm{T}}-3 \cos \theta_{\mathrm{D}} \cos \theta_{\mathrm{A}}\right)}{r^{3}}
$$

$$
\kappa^{2}=\left(\cos \theta_{\mathrm{T}}-3 \cos \theta_{\mathrm{D}} \cos \theta_{\mathrm{A}}\right)^{2}
$$

The orientation of the transition dipole moment of PCBs was determined by referring to Yang et $\mathrm{al}^{53}$ (Extended Data Figs. 10 and 11).

Absorption spectroscopy. Absorption spectra of the thylakoid membrane, PSII, PSII, and PBS were measured at $23^{\circ} \mathrm{C}$ using a UV-2600 spectrophotometer (Shimadzu, Kyoto, Japan). The spectrum of the thylakoid membrane was measured using the opal-glass method. separated on a SuperSep gel containing 10-20\% acrylamide (Wako, Tokyo, Japan) and then stained with Coomassie Blue (Extended Data Fig. 2). A gel imaging system (ChemiDoc 
540 polypeptides (a band containing $\mathrm{ApcC}$ and $\mathrm{CpcD}$ ) were identified by mass spectrometry. The

541 obtained protein bands were treated by in situ digestion using trypsin ${ }^{54}$. The fragmented peptides

542 were analyzed by peptide mass fingerprinting (PMF) and MS/MS using Autoflex speed (Bruker

543 Daltonics). The obtained mass spectra were analyzed by MASCOT server (MATRIX

544 SCIENCE).

Phylogenic analysis.

547 Thirty-eight sequences of ApcE from selected strains of cyanobacteria, glaucophytes, and

548 rhodophytes were obtained by blastp program at NCBI website

549 (https://blast.ncbi.nlm.nih.gov/Blast.cgi) (Extended Data Figs. 8 and 9). The sequences were

550 aligned using mafft ver. 7.478 with L-INS-i option ${ }^{55}$. The maximum likelihood tree of the ApcE

551 sequences were estimated using iqtree 2 ver. 2.1.4 with $\mathrm{LG}+\mathrm{F}+\mathrm{R} 5$ model selected by

552 ModelFinder. The statistical support of the trees was estimated with 1000 replications of ultrafast

553 bootstrap approximation ${ }^{56}$. Domain organization of ApcE was determined using hmmscan

554 program in HMMER ver. 3.3.2 (http://hmmer.org/) with Pfam database ${ }^{57}$. Phylogenetic tree and

555 domain organization were visualized using iToL ver. $4^{58}$. 


\section{Methods References}

41. Shen, J.-R., Kawakami, K. \& Koike, H. Purification and crystallization of oxygen-evolving photosystem II core complex from thermophilic cyanobacteria. Photosynth. Res. Protoc. 684, 41-51 (2011).

42. Kawakami, K. \& Shen, J. R. Purification of fully active and crystallizable photosystem II from thermophilic cyanobacteria. Methods Enzymol. 613, 1-16 (2018).

43. Zhang, J. et al. JADAS: A customizable automated data acquisition system and its application to ice-embedded single particles. J. Struct. Biol. 165, 1-9 (2009).

44. Zheng, S. Q., Palovcak, E., Armache, J. P., Verba, K. A., Cheng, Y. \& Agard, D. A. MotionCor2: anisotropic correction of beam-induced motion for improved cryo-electron microscopy. Nat. Methods. 14, 331-332 (2017).

45. Rohou, A. \& Grigorieff, N. CTFFIND4: fast and accurate defocus estimation from electron micrographs. J. Struct. Biol. 192, 216-221 (2015).

46. Zivanov, J., Nakane, T. \& Scheres, S. H. W. Estimation of high-order aberrations and anisotropic magnification from cryo-EM data sets in RELION-3.1. IUCrJ. 7, 253-267 (2020).

47. Punjani, A., Rubinstein, J. L., Fleet, D. J. \& Brubaker, M. A. cryoSPARC: algorithms for rapid unsupervised cryo-EM structure determination. Nat. Methods. 14, 290-296 (2017).

48. Zhang, K. Gctf: real-time CTF determination and correction. J. Struct. Biol. 193, 1-12 (2016).

49. Bell, J. M., Chen, M., Durmaz, T., Fluty, A. C. \& Ludtke, S. J. New software tools in EMAN2 inspired by EMDatabank map challenge. J. Struct. Biol. 204, 283-290 (2018). 
50. Rohou, A., Grant, T. \& Grigorieff, N. cis TEM, user-friendly software for single-particle image processing. Elife. 7, e35383 (2018).

51. Moriarty, N. W., Grosse-Kunstleve, R. W. \& Adams, P. D. Electronic Ligand Builder and Optimization Workbench (eLBOW): a tool for ligand coordinate and restraint generation. Acta Crystallogr. Sect. D Biol. Crystallogr. 65, 1074-1080 (2009).

52. Ramírez-Aportela, E. et al. FSC-Q: a CryoEM map-to-atomic model quality validation based on the local Fourier shell correlation. Nat. Commun. 12, 1-7 (2021).

53. Y. Yang, Y. et al. Active and silent chromophore isoforms for phytochrome Pr photoisomerization: An alternative evolutionary strategy to optimize photoreaction quantum yields. Struct. Dyn. 1 (2014).

54.Hellman, U., Wernstedt, C., Góñez, J. \& Heldin, C. H. Improvement of an "in-gel” digestion procedure for the micropreparation of internal protein fragments for amino acid sequencing. Anal. Biochem. 224 (1995), pp. 451-455.

55.Katoh, K. \& Standley, D. M. MAFFT multiple sequence alignment software version 7: Improvements in performance and usability. Mol. Biol. Evol. 30, 772-780 (2013).

56. Nguyen, L. T., Schmidt, H. A., Von Haeseler, A. \& Minh, B. Q. IQ-TREE: A fast and effective stochastic algorithm for estimating maximum-likelihood phylogenies. Mol. Biol. Evol. 32, 268-274 (2015).

57. Kalyaanamoorthy, S., Minh, B. Q., Wong, T. K. F., Von Haeseler, A. \& Jermiin, L. S. ModelFinder: Fast model selection for accurate phylogenetic estimates. Nat. Methods. 14, 587-589 (2017).

58. Hoang, D. T., Chernomor, O., Von Haeseler, A., Minh, B. Q. \& Vinh, L. S. UFBoot2: Improving the ultrafast bootstrap approximation. Mol. Biol. Evol. 35, 518-522 (2018). 
Acknowledgments: We thank research assistants Ms. Yuko Kageyama (Biostructural their help with cell cultures, preparation of samples, electrophoresis analysis, and spectroscopies.

612 We also thank Ms. Tomomi Shimonaka at the Graduate School of Science, Osaka City

613 University, for performing MS/MS spectrometry.

614 Funding: This work was supported by the Japan Society for the Promotion of Science (JSPS)

615 (JP20H05109 (to KK), JP20K06528 (to KK), and JP17H06434 (to NK)) and partly by the Joint 616 Usage/Research by Institute of Industrial Nanomaterials, Kumamoto University. JST-Mirai

617 Program Grant Number JPMJMI20G5 (to KY), and the Cyclic Innovation for Clinical

618 Empowerment (CiCLE) from the Japan Agency for Medical Research and Development, AMED 619 (to KK, TH and KY).

Author contributions: KK and NK designed the study; KK prepared the samples and performed electrophoresis analysis; KK measured spectroscopies, TH measured EM micrographs, and TH and KK processed the EM data (PBS core: TH, PC rod: KK) and reconstructed the final EM map

623 (PBS core: TH, PC rod: KK); KK performed structural analysis; YH performed phylogenetic tree analysis; DK commented on data analyses; KY, NK, and MM supervised this project; KK, TH, and $\mathrm{KY}$ wrote the draft manuscript; $\mathrm{KK}$, $\mathrm{TH}$, and $\mathrm{KY}$ revised the final manuscript, and all of the authors contributed to the interpretation of the results and improvement of the manuscript.

627 Competing interests: Authors declare that they have no competing interests.

628 Funding: This work was supported by the Japan Society for the Promotion of Science (JSPS) (JP20H05109 (to KK), JP20K06528 (to KK), and JP17H06434 (to NK)) and partly by the Joint 630 Usage/Research by Institute of Industrial Nanomaterials, Kumamoto University. JST-Mirai 
631 Program Grant Number JPMJMI20G5 (to KY), and the Cyclic Innovation for Clinical

632 Empowerment (CiCLE) from the Japan Agency for Medical Research and Development, AMED

633 (to KK, TH and KY).

634 Data and materials availability: Atomic coordinates and cryo-EM maps for the reported

635 structure of the PBS core and PC rod from Thermosynechococcus vulcanus were deposited in the

636 Protein Data Bank under accession codes 7VEA (PBS core) and 7VEB (PC rod), and in the

637 Electron Microscopy Data Bank under accession codes EMD-31944 (PBS core) and EMD-

63831945 (PC rod), respectively. Other data are available from the corresponding authors upon

639 reasonable request.

640 